\title{
BOEKWERKEN OP HET GEBIED VAN DE PLANTENVEREDELING
}

Books dealing with plant breeding

\section{H. DE HAAN}

Akerman, A., e.a. Svalöf 1886-1946. History and present problems. Carl Bloms Boktryckeri, Lund. 1948,389 pp., 79 fig.

BANGA, O., Veredeling van tuinbouwgewassen. Tjeenk Willink, Zwolle. 1944, 211 pp., 33 fig.

Chandrasegharan, S. N. and Parthasarathy, S. V. Cytogenetics and Plant Breeding. P. Varadachary \& Co, Madras. 1948, XVI and 589 pp., 156 fig.

Frimmel, Fr., Die Praxis der Pflanzenzüchtung. Paul Parey, Berlin. 1951, 159 pp., 13 fig.

Hagedoorn, A. L., Plant Breeding. Crosby Lockwood \& Son, London. 1950, 237 pp., 10 fig., 4 pl.

HAYES, H. K. and IMMER, F. R., Methods of plant breeding. McGraw-Hill Company, New York and London, 1942, 432 pp., 37 fig.

Hunter, H. and LEAKE, H. M., Recent advances in agricultural plant breeding. J. \& A Churchill, London. 1933, 361 pp., $16 \mathrm{pl}$.

KAPPERT, H., Die vererbungswissenschaftlichen Grundlagen der Pflanzenzüchtung. Paul Parey, Berlin und Hamburg. 1948, 244 pp., 79 fig.

KuCKuCK, H., Pflanzenzüchtung. Sammlung Göschen 1134, de Gruyter \& Co, Berlin. 1939, 125 pp., 12 fig.

KuckucK, H. und Mudra, A., Lehrbuch der allgemeinen Pflanzenzüchtung. S. Hirzel Verlag, Stuttgart. 1950.280 pp., 57 fig.

LAthouwers, V., Manuel de l'amélioration des plantes cultivées. II. L'Amélioration du froment. J. Ducolot, Gembloux. 1942, 579 pp., 67 fig.

LaWrence, W. J. C., Practical plant breeding. G. Allen \& Unwinn, London. 1945, 155 pp., 34 fig.

LINDQUist, B., Genetics in Swedish Forestry Practice. Svenska Skogsvardsföreningers Förlag, Stockholm, 1948, 174 pp., 65 fig.

ReEs, H. van, (2e druk bewerkt door Wiersema, H. T.) Veredelingsleer. Tjeenk Willink, Zwolle. 1949,110 pp., 18 fig.

ReINöHL, Fr., Pfianzenzüchtung. Ferd. Rau, Öhringen. 1939, 200 pp., 125 fig.

RichHaria, R. H., Plant Breeding and Genetics in India. Law Press, Patna. 1945. 403 pp.

Roemer, TH. und Rudorf, W., e.a., Handbuch der Pflanzenzüchtung. Paul Parey, Berlin und Hamburg. 1938-1950, 5 Bände.

SCHEIBE, A., Einführung in die Allgemeine Pflanzenzüchtung. Eugen Ulmer, Stuttgart. 1951, 475 pp., 122 fig.

SchiEMANN, E., Entstehung der Kulturpflanzen. Gebr. Bornträger, Berlin. Handbuch der Vererbungswissenschaft 3, Lief. 15, 1932, 377 pp., 96 fig.

Sengbusch, R. von, Theorie und Praxis der Pflanzenzüchtung. Societäts-Verlag, Frankfurt a.M.. 1939,127 pp., $35 \mathrm{fig}$.

VAVILOV, N. I., The origin, variation, immunity and breeding of cultivated plants (Transiated from the Russian by K. Starr Chester). Chronica Botanica 13, no 1-6, 1949-1950, 364 pp., 37 fig.

WeLlensieK, S. J., e.a., Grondslagen der algemene plantenveredeling. H. D. Tjeenk Willink \& Zoon, Haarlem. 1947, 541 pp., 90 fig.

Yearbook of Agriculture. Washington. 1936 (1189 pp.), 1937 (1487 pp.).

Het ligt in de bedoeling van de Redactie om in het vervolg een korte bespreking te geven van nieuwe boekwerken, voorzover deze ter recensie worden aangeboden.

\section{SUMMARY}

In the foregoing survey a few books dealing with plant breeding have been mentioned. It is the intention to review new books that have been submitted for criticism to the editing committee. 